\title{
The Analytical Study of Stator Tooth Modulation on Electromagnetic Radial Force in Permanent Magnet Synchronous Machines
}

\author{
Wenyi Liang, Jianfeng Wang, Patrick Chi-Kwong Luk, Senior Member, IEEE, Weizhong Fei, Member, IEEE,
}

\begin{abstract}
The electromagnetic radial force acting on the stator inner periphery will induce radial vibration and acoustic noise in permanent magnet synchronous machines. The radial force components are transmitted through the stator teeth to the yoke to introduce deformations. The influence of the stator tooth structure can be considered as an equivalent mechanical modulation effect on these electromagnetic radial force components. As a result, high-order electromagnetic radial force components can be modulated and potentially result in eminent stator low-mode vibration. In this paper, an analytical model is developed to offer an intuitive knowledge of stator tooth modulation effect on electromagnetic radial force. The validity of the proposed analytical method has beehE thiferanse pinned by both finite element analysis and experifiestitall results. Such an effective yet simple analytical model can be of significant benefit for the stator radial vibration analysis. It can be employed to not only promptly investigate the stator radial vibration characteristics but also perform effective optimization on stator radial vibration reduction in permanent magnet synchronous machines.
\end{abstract}

Index Terms-permanent magnet synchronous motor, electromagnetic radial force, tooth modulation effect, vibration.

\section{INTRODUCTION}

$\mathbf{T}$ HE acoustic noise and vibration, which are the main parasitic effects in permanent magnet synchronous machine (PMSM) drives, have been drawing particular attention throughout their design and operation stages. Electromagnetic radial force density (ERFD) is one of the primary sources for noise and vibration in low- to medium-power rated PMSMs [1]. Such noise and vibration associated with ERFD usually prevail in electric machines with low-speed condition [2]. The ERFD components in machine originates from the interaction of the magnetic flux density components. The stator slot and

Manuscript received March 12, 2020; revised June 14, 2020 and August 28,2020; accepted September 16,2020. Date of publication xxxx Xx,2020; date of current version Xxxx xx, 2020.(Corresponding author: Patrick Chi-Kwong Luk.)

Wenyi Liang, Jianfeng Wang, Weizhong Fei are with Hangzhou Branch of Zhejiang Tongxing Technology Co., Ltd., Hangzhou 310053 China. (e-mail: wenyi.liang@hotmail.com; wangjf1977@foxmail.com; achimao@hotmail.com)

Patrick Chi-Kwong Luk is with the Centre of Power Engineering, Cranfield University, Cranfield, MK43 OAL, U.K. (e-mail: p.c.k.luk@cranfield.ac.uk.

Color versions of one or more of the figures in this article are available online at http://ieeexplore.ieee.org.

Digital Object Identifier xx.xxxx/TIE.xxxx.xxxxxxx rotor pole number combination,and stator armature winding configuration play quite important roles to constitute air-gap magnetic field in PMSMs. The impacts of these two factors on the ERFD and its associated vibration are comprehensively investigated for inner-rotor PMSMs with fractional-slot and concentrated-winding (FSCW) configuration [3]-[6]. Besides, ERFD components have been also derived and investigated in rotor synchronous reference frame for their outer-rotor counterparts [7]-[10]. Moreover, various analytical [1], [11][13] and numerical [14]-[18] approaches have been proposed and widely employed to predict the vibration characteristics associated with ERFD in PMSMs. These ERFD components act on the stator tooth surfaces and the resultant force components are transmitted through the teeth to the stator yoke to cause deformations. The magnetic and mechanical anisotropy of stator lamination core caused by the slots has substantial effect on the force waves acting on the yoke. Most of the existing literatures as aforementioned solely adopt the stator slot permeance harmonic components to formulate the complex stator tooth effect on ERFD characteristics in PMSMs. Such simplification can potentially induce large errors and invalidate the predictions. Instead, the resultant ERFD components acting on each individual stator tooth are employed to predict the corresponding vibration characteristics in PMSMs [19]-[22]. However, the influence of the stator tooth structure can be considered as an equivalent mechanical modulation on the ERFD components. The demonstration and comprehensive analysis of such modulation effect in induction machine dates back to nearly 40 years ago [23].The recent revival of stator tooth modulation effect on electromagnetic radial force has been based on PMSMs with both FSCW [24] and integralslot configurations [25]. Additionally, the noticeable effect of local tangential force on vibration performance in FSCW PMSM has been investigated [26]. Generally, the resultant force components on the stator teeth are contributed by ERFD components with different spatial and temporal orders, and the tooth effects on these force components vary according to their spatial orders. The existing methods cannot provide the intrinsic relationship between the stator tooth configuration such as slot number, geometric shape, and ERFD characteristics. Consequently, it is of particular importance to develop a general analytical model, which can intuitively describe the stator tooth effect on the overall ERFD characteristics with reasonable accuracy.

In this study, a general analytical model is developed to 


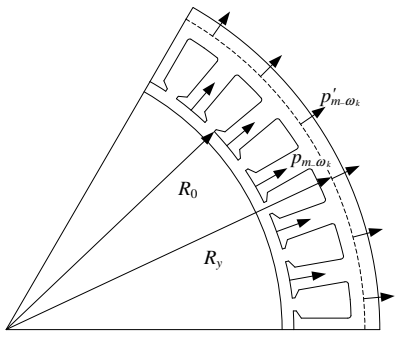

(a)

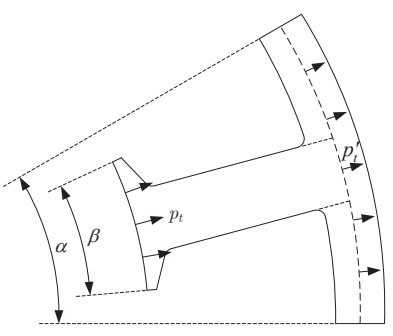

(b)

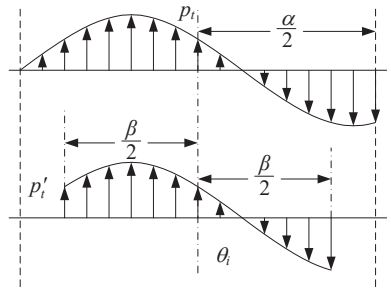

(c)

Fig. 1. Radial force acting on stator yoke: (a) existing model, (b) tooth effect model, (c) ERFD transmission model.

promptly evaluate the stator tooth effect on ERFD characteristics, whose accuracy is validated by both numerical and experimental results. The analytical derivations, which take into account the impact of stator tooth geometric parameters, can intuitively reveal the critical ERFD components and their main impact factors introduced by the stator tooth modulation effect. The proposed analytical model, together with the electromagnetic radial vibration analysis procedure, can be easily applied to all types of PMSMs. In Section II, the general theoretical model of stator radial vibration is introduced and followed by the analytical derivations of stator tooth modulation effect. Section III is dedicated to the application of the proposed analytical model on two PMSM prototypes with different configurations with numerical validations from finite element analysis (FEA) results, followed by comprehensive experimental analysis in Section IV. Section V provides the concluding remarks.

\section{Analytical Model of Tooth Modulation Effect}

\section{A. The Vibration of the Stator}

Strictly speaking, both the radial and tangential radial force components may excite radial vibration in the stator. Since the radial component is approximately an order of magnitude larger than the tangential counterpart in general, it is reasonable to neglect the contribution from the tangential components in stator radial vibration analysis [27]. Without loss of generality, the coupling in the direction between the radial and tangential directions are neglected and merely the ERFD components are considered in the proposed analytical model.

Generally, each ERFD component on the stator core inner peripheral can be expressed as

$$
p_{m_{-} \omega_{k}}\left(\theta_{m}, t\right)=P_{m_{-} \omega_{k}} \cos \left(m \theta_{m}-\omega_{k} t-\varphi_{m \_} \omega_{k}\right)
$$

where $P_{m \_\omega_{k}}, \omega_{k}, m$ and $\varphi_{m \_\omega_{k}}$ are the respective amplitude, angular frequency, spatial order and phase angle of the ERFD component. The amplitude of the introduced stator radial vibration displacement can be derived by [27]

$$
A_{m}=\frac{F_{m \_\omega_{k}}}{M_{s} \sqrt{\left(\omega_{m}^{2}-\omega_{k}^{2}\right)^{2}+4 \zeta^{2} \omega_{m}^{2} \omega_{k}^{2}}}
$$

where $M_{s}$ is the whole mass of the stator system. $\zeta$ is the corresponding modal damping ratio, and an empirical expression for the small sized electrical machine is suggested [28]

$$
\zeta=\frac{1}{2 \pi}\left(2.76 \times 10^{-5} f_{m}+0.062\right)
$$

and $F_{m_{-} \omega_{k}}$ can be expressed as

$$
F_{m \_\omega_{k}}=2 \pi R_{0} L_{e f} P_{m \_\omega_{k}}
$$

The stator system can be further simplified as a circular cylindrical shell with both ends free of constraints [27]. As a result, Equation 4 can also be rewritten in the terms of stator yoke radius instead as

$$
F_{m \_\omega_{k}}=2 \pi R_{0} L_{e f} P_{m \_\omega_{k}}^{\prime}
$$

$P_{m \_}^{\prime} \omega_{k}$ is the equivalent ERFD component acting on the yoke, as depicted in Fig.1a. Based on the geometry, it is normally obtained by

$$
P_{m \_\omega_{k}}^{\prime}=\frac{R_{0}}{R_{y}} P_{m \_\omega_{k}}
$$

\section{B. The Stator Tooth Modulation Effect Model}

The ERFD components can be directly derived from the interactions between all the magnetic flux density components in the air gap of the machine. In the existing conventional methods, the resultant air-gap ERFD components including slot opening regions are equivalently mapping to the stator yoke so that the resultant stator radial vibration can be predicted, as shown in Fig.1a. In reality, the stator inner peripheral is not continuous due to the slot openings and hence only the ERFD components in the stator tooth regions are considered in the proposed analytical method. The air-gap ERFD components acting on the stator tooth surfaces pass through the stator teeth to reach the stator yoke and cause deformation and vibration, as shown in Fig.1b. In order to maintain the force density vectorial synthesis conservative for each tooth, the equivalent ERFD components reaching the stator yoke cover the same arc width as the corresponding tooth as illustrated in Fig.1c.

As shown in Fig.1b and Fig.1c, the $i^{t h}$ tooth inner and top arc widths are $\alpha$ and $\beta$, and middle of the tooth is positioned at $\theta_{i}$. The corresponding amplitudes of the $n^{\text {th }}$ ERFD harmonic components on the stator yoke from the $i^{\text {th }}$ tooth, where $1 \leq$ $i \leq Z$ and $Z$ is the stator slot number, can be derived by 
applying the Fourier Series expansion of the tooth ERFD load as

$$
\left\{\begin{array}{l}
a_{n_{i}}=\frac{1}{\alpha} \int_{\theta_{i}-\alpha / 2}^{\theta_{i}+\alpha / 2} p_{m \_\omega_{k}}^{\prime}\left(\theta_{m}, t\right) \cos (n \theta) d \theta_{m} \\
b_{n_{i}}=\frac{1}{\alpha} \int_{\theta_{i}-\alpha / 2}^{\theta_{i}+\alpha / 2} p_{m_{-} \omega_{k}}^{\prime}\left(\theta_{m}, t\right) \sin (n \theta) d \theta_{m}
\end{array}\right.
$$

By substituting Equation (1) and Equation (6) into Equation (7), the amplitudes of the nth harmonic components from the $i^{\text {th }}$ tooth can be expressed as

$$
\left\{\begin{aligned}
a_{n_{i}}= & K_{m \_n} P_{m \_\omega_{k}}^{\prime} \\
& \cdot \cos \left((m \pm n)\left(\theta_{0}+(i-1) \alpha\right)-\omega_{k} t-\varphi_{m_{-} \omega_{k}}\right)(8) \\
b_{n_{i}}= & \pm K_{m \_n} P_{m \_\omega_{k}}^{\prime} \\
& \cdot \sin \left((m \pm n)\left(\theta_{0}+(i-1) \alpha\right)-\omega_{k} t-\varphi_{m \_\omega_{k}}\right)
\end{aligned}\right.
$$

where, $K_{m \_n}$ is determined by

$$
K_{m_{-} n}=\frac{Z}{(m \pm n) \pi} \sin \frac{(m \pm n) \beta}{2}
$$

By superposition of the components from all the stator teeth, the $n^{\text {th }}$ ERFD harmonic component introduced by $P_{m_{-} \omega_{k}}$ can be proposed as

$$
p_{n_{-} \omega_{k}}^{\prime}\left(\theta_{m}, t\right)=\sum_{i=1}^{Z}\left(a_{n_{i}} \cos \left(n \theta_{m}\right)+b_{n_{i}} \sin \left(n \theta_{m}\right)\right)
$$

By substituting Equation (8) into Equation (10), the harmonic component can be derived. Apparently, $p_{n \_\omega_{k}}^{\prime}=0$ when $m \pm$ $n \neq k Z, k$ is integer. With $m \pm n=k Z, p_{n_{-} \omega_{k}}^{\prime}$ can be represented as

$$
\begin{aligned}
p_{n \_\omega_{k}}^{\prime}\left(\theta_{m}, t\right)= & K_{m_{-} n} P_{m_{-} \omega_{k}}^{\prime} \\
& \cdot \cos \left(n \theta_{m} \pm \omega_{k} t \mp k Z \theta_{0} \pm \varphi_{m_{-} \omega_{k}}\right)
\end{aligned}
$$

and $K_{m \_n}$ in Equation (9) can be rewritten as

$$
K_{m \_n}=\frac{1}{k \pi} \sin \frac{k Z \beta}{2}
$$

Consequently, as a result of the tooth modulation effect, abundant of radial force components are introduced from the original $m^{\text {th }}$ order component with the new order of $n^{\text {th }}$. Noticeably, the rotational direction of the modulated ERFD component is opposite to the original component with $m+n=k Z$, while it keeps the same rotational direction with $m-n=k Z$. Normally, the slot opening coefficient is more frequently employed for the machine design so that Equation (12) can be rewritten as

$$
K_{m_{-} n}=\frac{1}{k \pi} \sin k K_{t} \pi
$$

where

$$
K_{t}=1-\frac{W_{b_{0}}}{W_{t}}
$$

where $K_{t}$ is slot opening coefficient, $W_{b_{0}}$ is the width of the slot opening, and $W_{t}$ is stator tooth pitch. According to Equation (13), there ought to be one or more slot opening coefficients which can minimize the modulated ERFD component of specific spatial order induced by certain original one. However, such optimal slot opening coefficients are
TABLE I

Structure SPECIFICATIONS OF PROTOTYPE PMSMS

\begin{tabular}{||c|c|c||}
\hline \hline Parameter & First Prototype & Second Prototype \\
\hline Pole pairs number & 3 & 5 \\
\hline Slot number & 9 & 12 \\
\hline Axial active length & $54 \mathrm{~mm}$ & $70 \mathrm{~mm}$ \\
\hline Stator inner diameter & $46 \mathrm{~mm}$ & $41 \mathrm{~mm}$ \\
\hline Stator outer diameter & $82 \mathrm{~mm}$ & $76 \mathrm{~mm}$ \\
\hline Stator yoke height & $4.5 \mathrm{~mm}$ & $3 \mathrm{~mm}$ \\
\hline Air-gap height & $0.75 \mathrm{~mm}$ & $0.5 \mathrm{~mm}$ \\
\hline Magnet height & $9 \mathrm{~mm}$ & $2 \mathrm{~mm}$ \\
\hline Pole arc coefficient & 1.0 & --- \\
\hline Magnet width & --- & $10.3 \mathrm{~mm}$ \\
\hline Stator mass & $0.75 \mathrm{~kg}$ & $1.24 \mathrm{~kg}$ \\
\hline \hline
\end{tabular}

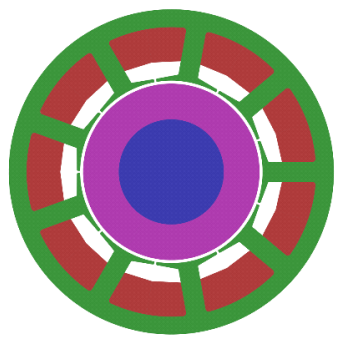

(a)

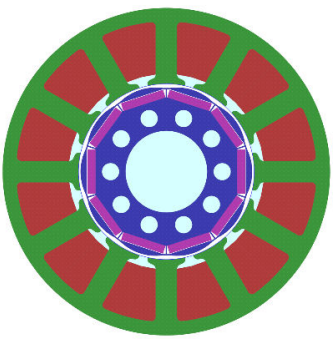

(b)
Fig. 2. Cross sections of prototype machines: (a) 9-slot-6-pole, (b) 12 slot-10-pole.

heavily influenced by the stator slot and rotor pole number combination.

It can be inspected from Equation (12) that the slot opening coefficient is the only parameter that will influence the tooth modulation effect. Therefore, the radial vibration of the stator shell can be effectively reduced by optimize the slot opening coefficient.

\section{Application of Stator ToOth Modulation EFFECT MODEL}

\section{A. PMSM Prototypes}

It can be observed from the analytical model in above section that stator tooth modulation effect plays a much more important role in PMSM with FSCW configuration than its integral-slot counterpart due to abundant interharmonic components. Consequently, two distinct FSCW PMSM prototypes are employed to implement the proposed analytical model for validation.The first prototype possesses 9 stator slots and 6 surface-mounted ferrite magnet poles, while the second one has 12 stator slots and 10 interior rare earth magnet poles. The main parameters of the two prototypes are given and compared in Table I, and the cross sections are depicted in Fig.2. Although the two prototypes have quite different axial lengths, they share very similar stator inner and outer diameters. The first prototype owns very small slot opening in order to minimize stator slot permeance harmonic components, and the analytical investigation of stator radial vibration for this machine is only based on no-load condition. Whilst the second prototype has relatively large slot opening and interior 


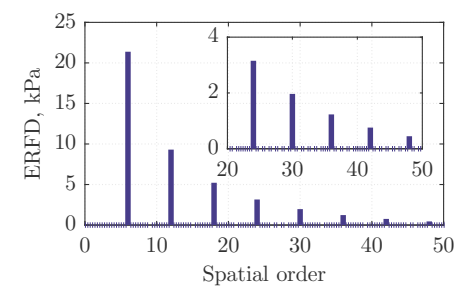

(a)

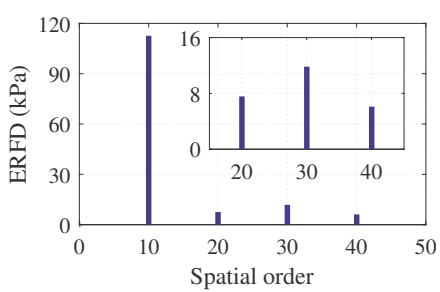

(b)
Fig. 3. No-load ERFD spectra in two PMSM prototypes: (a) first prototype, (b)second prototype.

magnet configuration to maximize the armature reaction effect, and the analytical investigation of stator radial vibration is based on no-load and flux-weakening (with high armature current) conditions.

\section{B. ERFD analysis and discussion}

The air-gap magnetic field components of the PMSM prototypes under different operational conditions can be directly obtained from two-dimensional (2-D) FEA models. The airgap ERFD components, which is introduced by the magnetic field components, can be directly evaluated from the dot product.

1) First Prototype: The corresponding ERFD components in the first prototype under no-load condition are evaluated and their spectra are compiled and given in Fig.3a. It is noteworthy that the temporal orders of these components are exactly the same as their spatial orders. It can be seen from the figure that the ERFD component with the lowest spatial order of $2 p=6$ is of the largest amplitude, where $p$ is the rotor pole pair number. This component mainly arises from the fundamental $(p=3)$ air-gap permanent magnet (PM) magnetic field. The ERFD component with spatial order of $4 p=12$ is $44 \%$, very close to half amplitude of the $2 p=6$ component, while the amplitude of $8 p=24$ order component can reach up to $15 \%$. Due to the tooth modulation effect, the third order shape deformation of the stator shell can be introduced by these components. Meanwhile, the frequencies of these components are several times of the fundamental component so that the associated accelerations on the stator yoke can be quite significant. As a result, all these components will be investigated with the mode of 3-order.

The ERFD components with spatial order higher than 40 will be neglected in further analysis due to their small amplitudes. On the other hand, there is a significant zeroth spatial order ERFD component, which is not included in Fig.3a. However, the corresponding frequency of this component is zero, thus it will only cause a static stator shell deformation but no vibration. Moreover, the component of $6 p=18$ and $12 p=36$ orders will introduce the zeroth order vibration with the angular frequencies of $6 \omega_{e}$ and $12 \omega_{e}$ due to the stator tooth modulation effect, where $\omega_{e}$ is the electric angular frequency. As a result, the zeroth modal analysis is required as well.

2) Second Prototype: The corresponding ERFD components in the first prototype under no-load condition are evaluated and their spectra are compiled and given in Fig.3b. Similarly, the temporal and spatial orders of these components

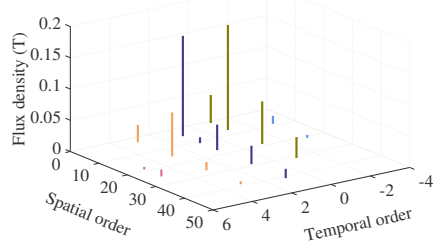

(a)

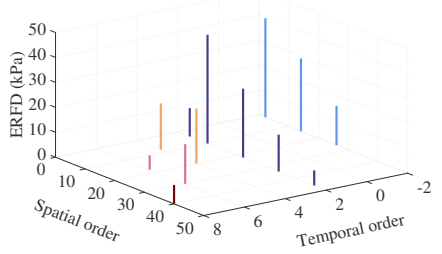

(b)
Fig. 4. ERFD analysis of second prototype under flux-weakening condition: (a) air-gap magnetic field components from armature reaction, (b) ERFD amplitudes.

TABLE II

The Mechanical Properties of Prototypes' material

\begin{tabular}{||c|c|c|c||}
\hline \hline Part & Material & Young's modulus & Poisson's ratio \\
\hline Stator & DW465-50 & $210 \mathrm{GPa}$ & 0.3 \\
\hline Shell & Aluminium & $70 \mathrm{GPa}$ & 0.3 \\
\hline Cover & Aluminium & $70 \mathrm{GPa}$ & 0.3 \\
\hline
\end{tabular}

are exactly same. Due to the eccentric rotor pole surface structure, the high harmonic components of the PM air-gap magnetic field are relatively small. As a result shown in Fig.3b, the ERFD component with spatial order of $2 p=10$ is the dominant component with a commanding amplitude while the rest ERFD components are one order of magnitude smaller. For the sake of comparison, the ERFD component with spatial order of $4 p=20$ is also employed for further radial vibration investigation. Moreover, the ERFD components with spatial order above 50 are all very small and hence neglected for further analysis. Due to its zero frequency, the large zeroth spatial order ERFD component is also ignored. According to the stator tooth modulation effect, stator radial vibration component with spatial order of $(2 p-Z)=2$ will be produced by the $2 p=10$ ERFD component, while the vibration component with spatial order of $(4 p-2 Z)=4$ originates from modulation of the $4 p=20$ ERFD component. The frequencies of those two components are $2 \omega_{e}$ and $4 \omega_{e}$, respectively.

On the other, the flux-weakening operation is implemented to achieve a high level armature phase current of $8 \mathrm{~A}$ and hence an evident armature reaction magnetic field. Assisted by the 2D transient electromagnetic FEA, comprehensive predictions of the main air-gap magnetic field components from the armature reaction under flux-weakening operation are obtained and its spatial and temporal spectra are complied and plotted in Fig.4a. Together with PM air-gap magnetic field components, the ERFD components with different spatial and temporal orders can be decoupled accordingly, and illustrated in Fig.4b. By comparison with Fig.3b, it can be inspected that more ERFD components appear under flux-weakening operation due to the interharmonic components from the armature reaction. Although all their amplitudes are lower than the $2 p=10$ component of no-load condition, more severe stator radial vibration tends to happen.

Noticeably, the rotational direction information of those airgap magnetic field components is embedded in the temporal order number. As a consequence, the spatial order numbers of the resultant ERFD components are always positive. The 


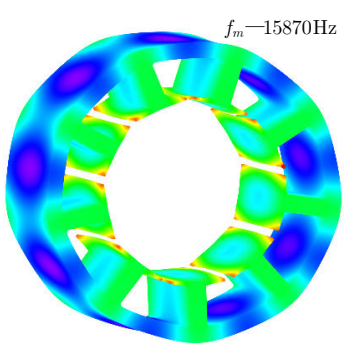

(a)

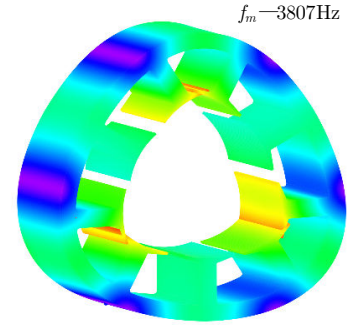

(b)
Fig. 5. Zeroth and third order modal shapes of first prototype: (a) zeroth mode, (b) third mode.

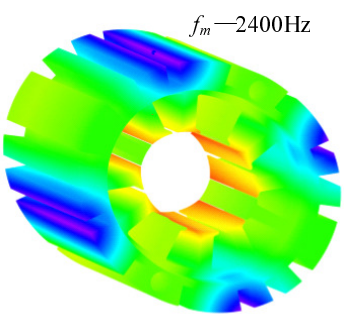

(a)

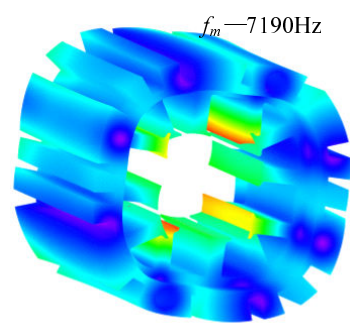

(b)
Fig. 6. Second and fourth order modal shapes of second prototype: (a) second mode, (b) fourth mode.

negative temporal order number represents opposite rotational direction against the fundamental component. It can be inspected from Fig. $4 \mathrm{~b}$ that the ERFD components with spatial orders of 2, 14 and 26, have quite evident amplitudes and the same frequency of $-2 \omega_{e}$. Based on the stator tooth modulation effect, stator radial vibration response with spatial order mode of 2 and frequency of $-2 \omega_{e}$ are introduced by the $14^{t h}$ and $26^{\text {th }}$ spatial order ERFD components. On the other hand, there are also remarkable ERFD components with spatial orders of 10, 22 and 34, and frequency of $2 \omega_{e}$. These components will also be modulated to induce stator radial vibration with spatial order mode of 2 but frequency of $-2 \omega_{e}$. All these stator radial vibration responses are with the same spatial order mode and frequency. They should be vectorially synthesized to obtain the resultant overall vibration. Analogously, the $8^{\text {th }}$ and $20^{\text {th }}$ spatial order ERFD components with frequency of $4 \omega_{e}$ will be modulated into the radial vibration components of a spatial order mode of 4 .

\section{Stator Natural Frequency Analysis}

The eigenvalues of the two prototype stator systems can be accurately obtained from modal analysis to facilitate the identification of the main ERFD components. The evaluated natural frequencies of different stator modes can be directly used for analytical stator radial vibration predictions. The material properties used in both prototypes for mechanical analysis are given in Table II.

1) First Prototype: Based on the above analysis, the $0^{\text {th }}$ and $3^{r d}$ spatial order harmonic components are the main cause of stator radial vibration in the first prototype machine under
TABLE III

THE ANALYTICAL AND NUMERICAL RESULTS OF FIRST PROTOTYPE

\begin{tabular}{||c|c|c|c|c||}
\hline \hline Condition & Mode & Frequency & Analytical & Numerical \\
\hline \multirow{4}{*}{ Noload } & 3 & 200 & $0.0265 \mathrm{~m} / \mathrm{s}^{2}$ & $0.0364 \mathrm{~m} / \mathrm{s}^{2}$ \\
\cline { 2 - 5 } & 3 & $400 \mathrm{~Hz}$ & $0.0465 \mathrm{~m} / \mathrm{s}^{2}$ & $0.0569 \mathrm{~m} / \mathrm{s}^{2}$ \\
\cline { 2 - 5 } & 0 & $600 \mathrm{~Hz}$ & $0.0064 \mathrm{~m} / \mathrm{s}^{2}$ & $0.0022 \mathrm{~m} / \mathrm{s}^{2}$ \\
\cline { 2 - 6 } & 3 & $800 \mathrm{~Hz}$ & $0.0635 \mathrm{~m} / \mathrm{s}^{2}$ & $0.0636 \mathrm{~m} / \mathrm{s}^{2}$ \\
\cline { 2 - 6 } & 3 & $1000 \mathrm{~Hz}$ & $0.0635 \mathrm{~m} / \mathrm{s}^{2}$ & $0.0201 \mathrm{~m} / \mathrm{s}^{2}$ \\
\cline { 2 - 6 } & 0 & $1200 \mathrm{~Hz}$ & $0.0059 \mathrm{~m} / \mathrm{s}^{2}$ & $0.0021 \mathrm{~m} / \mathrm{s}^{2}$ \\
\hline \hline
\end{tabular}

no-load condition. Modal analysis based on three-dimensional (3-D) mechanical FEA simulations are carried out to evaluate the $0^{t h}$ or $3^{r d}$ order natural frequencies of the stator system. The corresponding mode shapes of the stator are illustrated in Fig.5, which shows the natural frequencies of these two modes are $15870 \mathrm{~Hz}$ and $3807 \mathrm{~Hz}$ respectively. Fig.5b demonstrates distinctive third order mode characteristics of the stator. Meantime, noticeable sixth order mode characteristic of the stator yoke coexists due to the nine stator teeth. It can also be observed from Fig.5a that the coexistence of the zeroth and ninth order mode characteristics of the stator is quite evident. Although zeroth order mode is the lowest spatial order one, the corresponding eigenvalue is much larger than its third order counterpart. It implies that zeroth spatial order ERFD component will induce less significant vibration than other ones even with the same amplitude and frequency.

2) Second Prototype: From the previous analysis, the $2^{\text {nd }}$ and $4^{\text {th }}$ spatial order harmonic components are the main cause of stator radial vibration in the second prototype machine under both no-load and flux-weakening conditions. The $2^{\text {nd }}$ and $4^{\text {th }}$ order natural frequencies of the stator system are estimated by modal analysis based on 3-D mechanical FEA simulations, the resultant model shapes and frequencies are derived and depicted in Fig.6. It can be seen from the figure that the natural frequencies of the $2^{\text {nd }}$ and $4^{\text {th }}$ stator modes are $2400 \mathrm{~Hz}$ and $7190 \mathrm{~Hz}$, respectively.

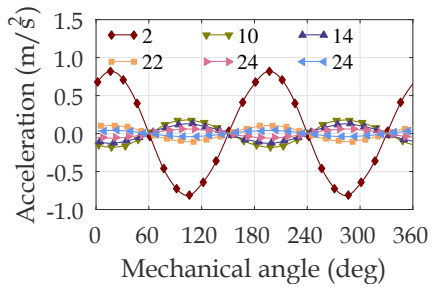

(a)

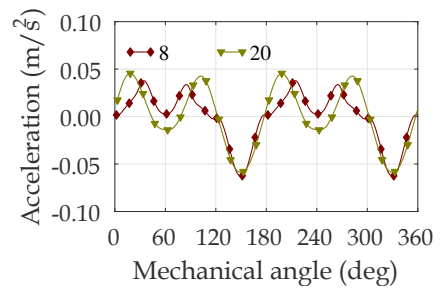

(b)
Fig. 7. Numerical vibration results of second prototype under fluxweakening condition: (a) $2 \omega_{e}$ frequency with different order components,(b) $4 \omega_{e}$ frequency with different order components.

\section{Numerical validation}

Based on the air-gap ERFD components from FEA evaluations above, the main ERFD components with 0th or 3rd spatial orders acting on the stator yoke of the first prototype are evaluated from Equation (11) and (14). The components with the same spatial and temporal orders are vectorially 
synthesized to feed into Equation (2) to (6), together with the natural frequencies of the stator. As a result, the main vibration results of the first prototype machine with no-load condition under the speed of 2000rpm are analytically evaluated and given in Table III. By the same measure, the analytical results of the second prototype machine with no-load and fluxweakening conditions under the same speed of 1200rpm are derived and listed in Table IV.

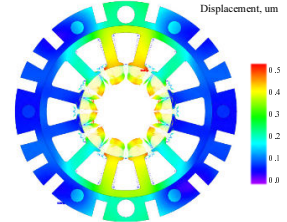

(a)

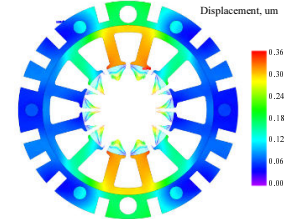

(b)

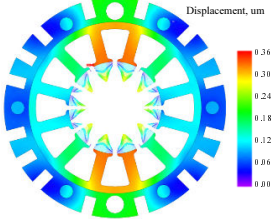

(c)
Fig. 8. Stator absolute displacement distributions of second prototype under flux-weakening condition: (a) with all force consideration, (b) without tangential force consideration, (c) without tangential force and slot region consideration.

In order to validate the analytical model, numerical analysis of the stator radial vibration has been carried out for the two prototype machines. The major ERFD components of the prototype machines are individually loaded on the stator tooth shoe surfaces in 3-D mechanical FEA models to gain the corresponding stator radial vibration responses. Moreover, the vectorial synthesis for the radial vibration responses with same spatial and temporal orders has been undertaken to achieve the overall results. The numerical stator radial vibration results for the first and second prototypes under no-load conditions can be directly obtained from FEA simulations without synthesization, and presented in Table III and IV, respectively. As aforementioned, the air-gap ERFD components with spatial orders of $2,10,14,22,26$, and 34 in the second prototype with flux-weakening condition can all be modulated to induce stator radial vibration response with spatial order mode of 2 and frequency of $-2 \omega_{e}$ and the numerical results are obtained and shown in Fig.7a. Similarly, the air-gap ERFD components with spatial orders of 8 and 20 in the second prototype with flux-weakening condition can induce stator radial vibration response with spatial order mode of 4 and frequency of $4 \omega_{e}$ and the numerical results are derived and depicted in Fig. $7 \mathrm{~b}$. The numerical results in Fig.7 are synthesized accordingly and demonstrated in Table IV.The analytical and numerical results of the main stator radial vibration for both prototype machines in Table III and IV show quite reasonably good agreements with maximum relative error of about $50 \%$. By considering the rather simplified vibration model in Equation 2, it is fair to conclude the proposed analytical model is validated by the numerical results.

Electromagnetic and mechanical coupling FEA simulations are carried out to reveal the stator displacements in the second prototype under flux-weakening condition so that the assumption made for the proposed method can be somewhat justified. Three scenarios, one with all electromagnetic force considered, one without electromagnetic tangential force considered, and the other without both electromagnetic tangential force and
TABLE IV

THE ANALYTICAL AND NUMERICAL RESULTS OF SECOND PROTOTYPE

\begin{tabular}{||c|c|c|c|c||}
\hline \hline Condition & Mode & Frequency & Analytical & Numerical \\
\hline \multirow{2}{*}{ Noload } & 2 & $200 \mathrm{~Hz}$ & $0.633 \mathrm{~m} / \mathrm{s}^{2}$ & $0.45 \mathrm{~m} / \mathrm{s}^{2}$ \\
\cline { 2 - 5 } & 4 & $400 \mathrm{~Hz}$ & $0.0158 \mathrm{~m} / \mathrm{s}^{2}$ & $0.0154 \mathrm{~m} / \mathrm{s}^{2}$ \\
\hline $\begin{array}{c}\text { Flux- } \\
\text { Weakening }\end{array}$ & 2 & $200 \mathrm{~Hz}$ & $0.739 \mathrm{~m} / \mathrm{s}^{2}$ & $0.599 \mathrm{~m} / \mathrm{s}^{2}$ \\
\cline { 2 - 5 } & 4 & $400 \mathrm{~Hz}$ & $0.0925 \mathrm{~m} / \mathrm{s}^{2}$ & $0.0782 \mathrm{~m} / \mathrm{s}^{2}$ \\
\hline \hline
\end{tabular}

slot region force considered, have been investigated and the corresponding stator absolute displacement distributions are depicted in Fig.8. Furthermore, the radial displacements over the stator core outer periphery are obtained and compiled in Fig.9, which reveals all three scenarios share the same displacement pattern with very close results. The method adopted in this study has slightly larger radial displacement than the one with slot region but electromagnetic tangential force consideration in the regions nearby 0 degree and 180 degree, while it possesses rather smaller radial displacement than the one with all force consideration in the regions nearby 90 degree and 270 degree. The assumptions in the proposed method drastically simplify the evaluation of stator tooth modulation on electromagnetic radial force in PMSMs and make the analytical approach practically feasible. Such simplifications indeed bring some prediction errors, however it is still of particular importance to have such analytical method with reasonable accuracy for preliminary assessment and optimization purposes.

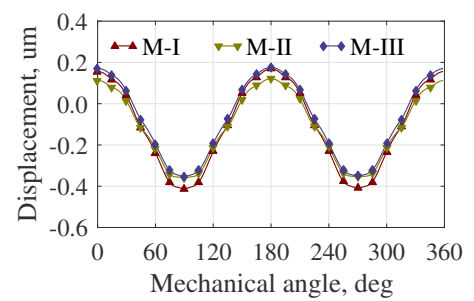

Fig. 9. The radial displacement of stator core outer periphery in second prototype under flux-weakening condition.(M-I:with all force consideration;M-II:without tangential force consideration;M-III:without tangential force and slot region consideration)

\section{E. Experimental Validation}

The experimental tests on the two prototype FSCW PMSM drive system under different operational conditions are carried out in order to further underpin the validity of the proposed analytical model in this section. The prototype PMSMs are driven by two-level voltage source inverter (VSI) with SVPWM technique, and the experimental setups are depicted as Fig.10. The mechanical transmission and load attached to the rotor shafts will impose inevitable changes on the mechanical characteristics of the prototypes, which can be very difficult to predict. Hence, the shaft of the prototype FSCW PMSMs are not connected to any mechanical transmissions or load equipments so that such uncertain interferences can be eliminated. The Dytran 3023A1 triaxial IEPE accelerometer is employed to measure the radial vibration of the prototype 


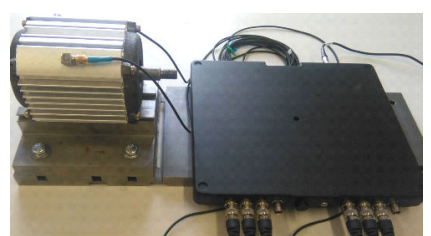

(a)

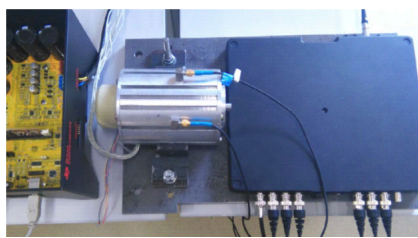

(b)
Fig. 10. The experimental setup of prototype PMSM drives: (a) first prototype,(b) second prototype.

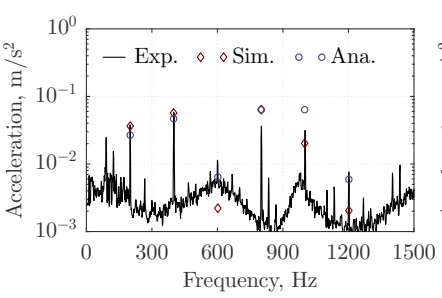

(a)

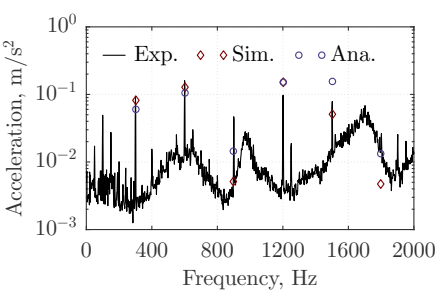

(b)
Fig. 11. Experimental validation of the first prototype: (a) vibration at 2000rpm,(b) vibration at 3000rpm.(Exp.-Experimental result, Sim.Simulation result, Ana.-Analytical result)

stator during the experiment, while Soundbook Expander 908011.6 from SINUS Messtechnik $\mathrm{GmbH}$ is used for data acquisition and process.

1) First Prototype: The experimental results of the first prototype with no-load conditions under speeds of 2000rpm and 3000rpm are obtained and compared with the corresponding analytical and numerical results in Fig.11. It can be seen from the figure that the analytical model can well reveal the stator radial vibration characteristics of the first prototype. Relatively severe radial vibrations occur at frequencies of $2 \omega_{e}, 4 \omega_{e}, 8 \omega_{e}$, and $10 \omega_{e}$, while they hardly exist at frequencies of $6 \omega_{e}$ and $12 \omega_{e}$. As the corresponding ERFD components with spatial orders of $2 p=6,4 p=12,8 p=24$ and $10 p=30$ can be modulated into third spatial order components, evident radial vibrations are induced accordingly on the stator yoke. On the other hand, the nearly closed-slot configuration makes tooth effect coefficients very close to zero for ERFD components with spatial orders of $6 p=18$ and $12 p=36$. As a result, the corresponding modulated ERFD components are insignificant. The small ERFD amplitudes, together with the large stator natural frequency of zeroth spatial order mode, result in trivial vibrations from such ERFD components. The analytical model slightly underestimates the $2 \omega_{e}$ and $4 \omega_{e}$ components and mildly overestimates the $8 \omega_{e}$ and $10 \omega_{e}$ components.

2) Second Prototype: The experimental test on the second prototype with no load connection under flux-weakening current of $8 \mathrm{~A}$ are carried out. The corresponding armature current waveform is captured and depicted as Fig.12a, while its spectra is also compiled and shown in Fig.12b. It can be observed from the figure that the armature phase current is essentially sinusoidal with negligible phase-belt harmonic distortion. The influence of the armature reaction on the stator radial vibration can be effectively demonstrated and investigated by such flux-weakening current. With a DC link voltage

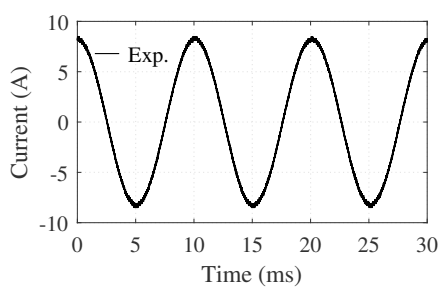

(a)

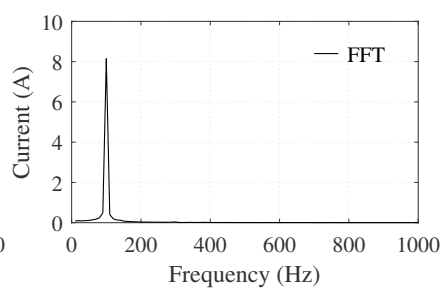

(b)
Fig. 12. Experimental armature current of the second prototype under flux-weakening condition at 1200rpm (a) current waveform,(b) current spectrum.

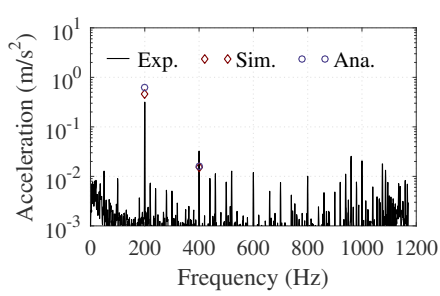

(a)

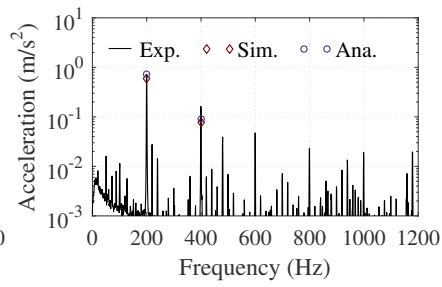

(b)
Fig. 13. Experimental validation of the Second prototype: (a) no-load condition,(b) flux-weakening condition.(Exp.-Experimental result, Sim.Simulation result, Ana.-Analytical result)

of $21 \mathrm{~V}$, the prototype with an $8 \mathrm{~A}$ flux-weakening current can reach the maximum speed of 1200rpm. Furthermore, the DC link voltage is increased to $60 \mathrm{~V}$ to achieve the same speed for the second prototype without load connection and fluxweakening current. Consequently, the stator radial vibration of the machine under different operations can be fairly compared.

The experimental results of stator radial vibration are obtained at the rotational speed of 1200rpm, and the spectra of the stator radial vibration for both conditions are compiled and depicted with analytical and numerical results in Fig.12. The figure shows the stator radial vibration with spatial order mode of 2 and frequency of $-2 \omega_{e}$ are the dominant components with both operational conditions. Generally, the analytical model provides very close predictions. It tends to slightly overestimate the stator radial vibration component with frequency of $-2 \omega_{e}$ in the prototype under no-load condition. The analytical model also accurately estimates this component under fluxweakening condition. It can be also observed that the model slightly underestimates the stator radial vibration components with frequency of $-4 \omega_{e}$ under both conditions. Moreover, the numerical method tends to slightly overestimate the stator radial vibration component with frequency of $-2 \omega_{e}$ under no-load condition. Whereas, it delivers a slight underestimate under flux-weakening condition. It can be also inspected that they also slightly underestimate the stator radial vibration components with frequency of $-4 \omega_{e}$ in the prototype under both conditions.

3) Discussions: Generally, the mechanical behaviours in the stator systems of the proposed two prototype machines are rather complex.It is practically impossible to analytically characterize such sophisticated phenomenon completely. Moreover, the modal damping ratios from Equation (3) are 
rather empirical so that inevitable errors can be induced. By taking into account all these factors, it is fair to conclude that the analytical results are in good agreements with the corresponding numerical and experimental results. Overall, the validity of the proposed analytical model of stator tooth modulation effect on stator radial vibration has been underpinned by the numerical and experimental results.

\section{CONCLUSION}

In this study, a simple yet effective analytical model is developed to account for the stator tooth modulation effect on ERFD components and hence the stator radial vibration in PMSM. Both surface-mounted and interior PMSMs are employed for the application of the proposed analytical model. Both numerical analysis and experimental tests are carried out to validate the proposed stator tooth modulation model. Based on the proposed stator tooth modulation effect model, the characteristics of stator radial vibration can be accurately predicted and effectively illustrated. Moreover, the influences of the design parameters on the stator radial vibration characteristics can also be insightfully revealed by the corresponding analytical model. The proposed method can be further employed to facilitate quick preliminary study on stator radial vibration optimization.

\section{REFERENCES}

[1] R. Islam and I. Husain, "Analytical model for predicting noise and vibration in permanent-magnet synchronous motors," IEEE Transactions on Industry Applications, vol. 46, no. 6, pp. 2346-2354, Nov 2010.

[2] B. M. Wilamowski and J. D. Irwin, Power Electronics and Motor Drives. CRC Press, 2016

[3] S. O. Kwon, J. J. Lee, T. Sun, and J. P. Hong, "Characteristics and radial magnetic force of interior permanent magnet synchronous motor according to pole/slot combinations," in 2009 IEEE Vehicle Power and Propulsion Conference, Sept 2009, pp. 1491-1495.

[4] Z. Q. Zhu, Z. P. Xia, L. J. Wu, and G. W. Jewell, "Influence of slot and pole number combination on radial force and vibration modes in fractional slot PM brushless machines having single- and double-layer windings," in 2009 IEEE Energy Conversion Congress and Exposition, Sept 2009, pp. 3443-3450.

[5] T. Sun, J. M. Kim, G. H. Lee, J. P. Hong, and M. R. Choi, "Effect of pole and slot combination on noise and vibration in permanent magnet synchronous motor," IEEE Transactions on Magnetics, vol. 47, no. 5, pp. 1038-1041, May 2011.

[6] I. Jang and W. Kim, "Study on electromagnetic vibration analysis process for PM motors," IEEE Transactions on Applied Superconductivity, vol. 30, no. 4, pp. 1-6, 2020.

[7] J. Hur, Y. D. Chun, J. Lee, and D. S. Hyun, "Dynamic analysis of radial force density in brushless DC motor using 3-D equivalent magnetic circuit network method," IEEE Transactions on Magnetics, vol. 34, no. 5, pp. 3142-3145, Sep 1998.

[8] H. C. M. Mai, R. Bernard, P. Bigot, F. Dubas, D. Chamagne, and C. Espanet, "Consideration of radial magnetic forces in brushless DC motors," in 2010 International Conference on Electrical Machines and Systems, Oct 2010, pp. 1-6.

[9] J. Krotsch and B. Piepenbreier, "Radial forces in external rotor permanent magnet synchronous motors with non-overlapping windings," IEEE Transactions on Industrial Electronics, vol. 59, no. 5, pp. 2267-2276, May 2012.

[10] Z. Shi, X. Sun, G. Lei, Z. Yang, Y. Guo, and J. Zhu, "Analysis and optimization of radial force of permanent-magnet synchronous hub motors," IEEE Transactions on Magnetics, vol. 56, no. 2, pp. 1-4, 2020

[11] S. Huang, M. Aydin, and T. A. Lipo, "Electromagnetic vibration and noise assessment for surface mounted PM machines," in 2001 Power Engineering Society Summer Meeting. Conference Proceedings, vol. 3, July 2001, pp. 1417-1426.
[12] J. F. Gieras, C. Wang, C. S. L. Joseph, and N. Ertugrul, "Analytical prediction of noise of magnetic origin produced by permanent magnet brushless motors," in 2007 IEEE International Electric Machines Drives Conference, vol. 1, May 2007, pp. 148-152.

[13] D. Braunisch, B. Ponick, and G. Bramerdorfer, "Combined analyticalnumerical noise calculation of electrical machines considering nonsinusoidal mode shapes," IEEE Transactions on Magnetics, vol. 49, no. 4 pp. 1407-1415, April 2013.

[14] T. Ishikawa, H. Inaba, and M. Matsunami, "Comparison of vibration characteristics of several interior permanent magnet synchronous motors," in 2008 International Conference on Electrical Machines and Systems, Oct 2008, pp. 314-319.

[15] H. Yang, Z. Han, and Y. Chen, "Electromagnetic vibration of interior permanent magnet brushless motors under brushless DC and AC operation," in 2009 International Conference on Electrical Machines and Systems, Nov 2009, pp. 1-6.

[16] D. Torregrossa, B. Fahimi, F. Peyraut, and A. Miraoui, "Fast computation of electromagnetic vibrations in electrical machines via field reconstruction method and knowledge of mechanical impulse response," IEEE Transactions on Industrial Electronics, vol. 59, no. 2, pp. 839-847, Feb 2012.

[17] K. H. Yim, J. W. Jang, G. H. Jang, M. G. Kim, and K. N. Kim, "Forced vibration analysis of an IPM motor for electrical vehicles due to magnetic force," IEEE Transactions on Magnetics, vol. 48, no. 11, pp. 2981-2984, Nov 2012.

[18] M. Boesing and R. W. De Doncker, "Exploring a vibration synthesis process for the acoustic characterization of electric drives," IEEE Transactions on Industry Applications, vol. 48, no. 1, pp. 70-78, Jan 2012.

[19] Y. S. Chen, Z. Q. Zhu, and D. Howe, "Vibration of PM brushless machines having a fractional number of slots per pole," IEEE Transactions on Magnetics, vol. 42, no. 10, pp. 3395-3397, Oct 2006.

[20] Y. Chen, H. Yang, and Z. Han, "Investigation of electromagnetic vibration of permanent magnet brushless machines," in 2008 International Conference on Electrical Machines and Systems, Oct 2008, pp. 621626.

[21] A. Cassat, C. Espanet, R. Coleman, L. Burdet, E. Leleu, D. Torregrossa, J. M'Boua, and A. Miraoui, "A practical solution to mitigate vibrations in industrial PM motors having concentric windings," IEEE Transactions on Industry Applications, vol. 48, no. 5, pp. 1526-1538, Sept 2012.

[22] M. S. Islam, R. Islam, and T. Sebastian, "Noise and vibration characteristics of permanent-magnet synchronous motors using electromagnetic and structural analyses," IEEE Transactions on Industry Applications, vol. 50, no. 5, pp. 3214-3222, Sept 2014.

[23] B. Wachta, "Influence of stator slots on the development of noisegenerating magnetic force waves," Siemens Forschungs-und Entwicklungsberichte, 1981.

[24] H. Fang, D. Li, R. Qu, and P. Yan, "Modulation effect of slotted structure on vibration response in electrical machines," IEEE Transactions on Industrial Electronics, vol. 66, no. 4, pp. 2998-3007, 2019.

[25] S. Wang, J. Hong, Y. Sun, and H. Cao, "Analysis of zeroth-mode slot frequency vibration of integer slot permanent-magnet synchronous motors," IEEE Transactions on Industrial Electronics, vol. 67, no. 4, pp. 2954-2964, 2020.

[26] H. Lan, J. Zou, Y. Xu, and M. Liu, "Effect of local tangential force on vibration performance in fractional-slot concentrated winding permanent magnet synchronous machines," IEEE Transactions on Energy Conversion, vol. 34, no. 2, pp. 1082-1093, 2019.

[27] J. F. Gieras, C. Wang, and J. C. Lai, Noise of Polyphase Electric Motors, ser. Electrical and Computer Engineering. CRC Press, 2005. [Online]. Available: https://books.google.co.uk/books?id=SUVuBwAAQBAJ

[28] S. J. Yang, Low-Noise Electrical Motors, ser. Monographs in Electrical and Electronic Engineering. Oxford University Press, 1981. 


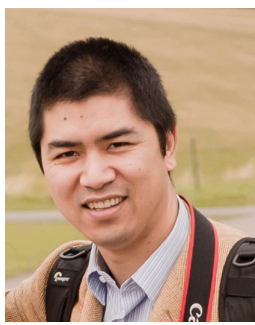

Wenyi Liang was born in Zhejiang, China, 1982. $\mathrm{He}$ received the B.Eng. and M.Eng. degrees in electrical engineering from Zhejiang University, Hangzhou, China, in 2004 and 2006, respectively.

From 2006 to 2014, he was a Development Engineer in Hangzhou Easitech Corporation, Hangzhou. He is currently a Research Fellow with the Power Engineering Centre, Cranfield University, Cranfield, U.K. His current research interests include design, analysis, and applications of electric machines and drives.

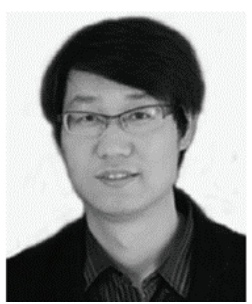

Jianfeng Wang Jianfeng Wang was born in Taiyuan, China, 1977. He received the B.Eng. and M.Eng. degrees in electrical engineering from Taiyuan University of Technology, Taiyuan, China, in 1999 and 2003, respectively.

From 2003 to 2007, he was a Lecturer with the Department of Electric Engineering, Taiyuan University of Technology, Taiyuan, China. From 2007 to 2015, he had been with Hangzhou Easitech Corporation, Hangzhou, China and had served as CTO for Hangzhou Fusheng Electrical Appliance Co.,Ltd. which is wholly-owned subsidiary of Shanghai Highly (Group) Co.,Ltd. (2015-2017). His current research interests include design, applications, and production process in electric machines.

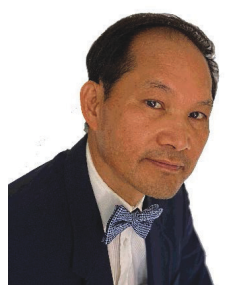

Patrick Chi-Kwong Luk Patrick Chi-Kwong LUK was born in Hong Kong. He received the High Diploma with Merits (BSc) from Hong Kong Polytechnic University (PolyU), in 1983, the M.Phil degree from Sheffield University, U.K., in 1989, and the Ph.D. degree from the University of South Wales, U.K., in 1992, all in electrical engineering.

He started his careers in industry as Engineer Trainee between 1981 and 1983 at GEC (H.K.) and then as Applications Engineer at Polytek Engineering Co. (H.K.) after graduation. In 1986, he had the first taste of a research career while working as a Research Associate in the Industrial Centre at PolyU. In 1987, with the award of a British Council scholarship, he moved to the U.K. to continue his research career in the academia, and had since held research/academic positions at the universities of Sheffield, South Wales and Hertfordshire, U.K. He joined Cranfield University, U.K., in 2002, where he is Chair Professor in electrical engineering. $\mathrm{He}$ is an active IEEE volunteer and had served as Chairman for IEEE UKI Power Electronics Chapter (20072016) and IEEE Professional Communications Society (2017- ) among other roles. $\mathrm{He}$ is Associate Editor for many flagship journals including IEEE Power Electronics. He has authored over 270 publications and several patents in electric machines and power electronics. His current research interests include power electronics and drives, electrification of transportation and energy systems.

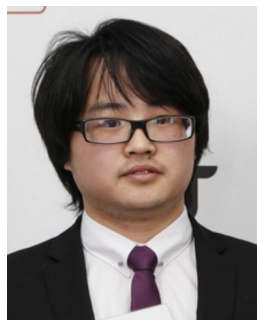

Weizhong Fei (M'12) was born in Zhejiang, China, 1981. He received the B.Eng. and M.Eng. degrees from Zhejiang University, Hangzhou, China, in 2004 and 2006, respectively, and the Ph.D. degree from Cranfield University, Shrivenham, U.K., in 2010, all in electrical engineering.

From 2011 to 2012, he was a Research Associate at the University of Sheffield, Sheffield, U.K. Since 2012, he has been with Cranfield University, Cranfield, U.K. where he is currently a Lecturer in Clean Energy Technology. His current research interests include design and applications of high efficient energy conversion systems. 